$m_{n}$, all other solutions being obtained from this solution by the addition of multiples of $k$.

THEOREM 2. If the minimum equations of $n$ finite square matrices $m_{1}$ to $m_{n}$ with elements in a field $F$ are relatively prime then for any set of $n$ polynomials $h_{1}, \cdots, h_{m}$, in $F$, a polynomial $f$ may be found such that

$$
f\left(m_{i}\right)=h_{i}\left(m_{i}\right), \quad(i=1, \cdots, n) .
$$

These theorems specialize to the above mentioned algebraic theorem since each $x_{i}$ is a one by one matrix with minimum equation $\lambda-x_{i}=0$.

It should be noted that in the above discussion no restriction as to the field in which the elements of the matrices may lie is made, nor are the $m_{i}$ necessarily of the same order.

The University of Wisconsin

\title{
PROBLEMS OF THE CALCULUS OF VARIATIONS WITH PRESCRIBED TRANSVERSALITY CONDITIONS*
}

\section{BY LINCOLN LA PAZ $\dagger$}

1. Introduction. Problems of the calculus of variations in the plane for which a prescribed relation exists between the directions of the extremals and the transversals were first studied by Stromquist $\ddagger$ and Bliss. § Recently Rawles, || using a method based on properties of the Hilbert invariant integral, has given an interesting treatment of the analogous problem in $\left(x, y_{1}, \cdots, y_{n}\right)$-space.

In the present paper the latter problem is attacked from a quite different point of view. The method here used avoids a restrictive hypothesis made by Rawles with regard to the ex-

* Presented to the Society, August 29, 1929.

$\dagger$ National Research Fellow, 1928-1929.

‡ Stromquist, Transactions of this Society, vol. 7 (1906), p. 181; Annals of Mathematics, (2), vol. 9 (1907), p. 57.

$\S$ Bliss, Annals of Mathematics, (2), vol. 9 (1907), p. 134.

II Rawles, Transactions of this Society, vol. 30 (1928), pp. 765-784.

9 The possibility of approaching the problem from this viewpoint was suggested to the writer by G. A. Bliss. 
istence of an $n$-parameter family of Mayer fields;* and the results of his paper are supplemented by a proof that his formula $f=g e^{H}$ actually gives the most general non-singular integrand function $f$ compatible with a prescribed transversality relation.

2. Properties of the Transversality Coefficients of a NonSingular Problem of the Calculus of Variations. For a problem of minimizing the integral

$$
I=\int_{x_{1}}^{x_{2}} f\left(x, y_{1}, \cdots, y_{n}, y_{1}^{\prime}, \cdots, y_{n}^{\prime}\right) d x
$$

an extremal $E$ through the point $\left(x, y_{1}, \cdots, y_{n}\right)$ in the direction $\left(1: y_{1}^{\prime}: \cdots: y_{n}^{\prime}\right)$ is said to be cut transversally by the hyperplane of directions $\left(d x: d y_{1}: \cdots: d y_{n}\right)$ through this point in case

$$
\left(f-y_{\mu}^{\prime} \partial f / \partial y_{\mu}^{\prime}\right) d x+\left(\partial f / \partial y_{\mu}^{\prime}\right) d y_{\mu}=0 .
$$

In (2) the arguments in $f$ and its derivatives are $x, y_{1}, \cdots, y_{n}$, $y_{1}^{\prime}, \cdots, y_{n}^{\prime}$ and, as elsewhere in this paper, a repeated Greek letter is an umbral index indicating a summation with the range 1 to $n$ unless otherwise specified. This definition of transversality is the usual one: it makes no appeal to the notion of a field or the transversal hypersurfaces thereof and defines what is meant by transversality for every set $\left(x, y_{1}, \cdots, y_{n}\right.$, $\left.y_{1}^{\prime}, \cdots, y_{n}^{\prime}\right)$ in the fundamental region $R$ of the integral (1) $\ddagger$.

If (1) is a non-singular problem, that is to say, if there exists a region $R$ in which $D=\left|\partial^{2} f / \partial y_{i}^{\prime} \partial y_{j}^{\prime}\right|,(i, j=1, \cdots, n)$, is everywhere different from zero, $\S$ then the quantity $\left(f-y_{\mu}^{\prime} \partial f / \partial y_{\mu}^{\prime}\right)$

* Rawles, loc. cit., p. 774.

$\dagger$ Loc. cit., p. 778.

$¥$ Consult in this connection Bliss, Calculus of Variations, 1925, p. 129. In the present paper we assume that $f$ is of class $C^{\prime \prime \prime}$ in a region $R$ of $\left(x, y_{1}, \cdots, y_{n}, y_{1}^{\prime}, \cdots, y_{n}^{\prime}\right)$-values. For a definition of the term class as here used see Bolza, Vorlesungen über Variationsrechnung, p. 13.

$\S$ Problems for which $D \not \equiv 0$ have been called regular by Goursat (Cours d'Analyse Mathématique, vol. III, 1923, p. 561) and ordinary by Hadamard (Lȩ̧ons sur le Calcul des Variations, vol. I, 1910, p. 68). The term nonsingular has been employed in lectures by G. A. Bliss, who characterizes a problem as regular only in case the quadratic form with matrix $\left\|\partial^{2} f / \partial y_{i}^{\prime} \partial y_{j}^{\prime}\right\|$ is positive definite. This latter usage seems preferable since it requires that in higher space as in the plane the Legendre condition in stronger form shall hold for regular problems. 
is not identically zero. For if $f$ satisfies the equation $f-y_{\mu}^{\prime} \partial f / \partial y_{\mu}^{\prime}=0$ it can be shown by consideration of the associated symmetric system

$$
d y_{1}^{\prime} / y_{1}^{\prime}=\cdots=d y_{n}^{\prime} / y_{n}^{\prime}=d f / f
$$

that the most general non-singular integral $f$ of $f-y_{\mu}^{\prime} \partial f / \partial y_{\mu}^{\prime}=0$ is given by

$$
\begin{gathered}
f=y_{1}^{\prime} F\left(u_{2}, \cdots, u_{n}, x, y_{1}, \cdots, y_{n}\right), \quad u_{k}=y_{k}^{\prime} / y_{1}^{\prime}, \\
k=2, \cdots, n,
\end{gathered}
$$

where $F$ is an arbitrary function of its arguments. But for the integrand function $f$ in (4) it is found that

$$
D=\left|\begin{array}{rrrr}
\frac{\partial^{2} F}{\partial u_{\beta} \partial u_{\gamma}} & \frac{y_{\beta}^{\prime} y_{\gamma}^{\prime}}{\left(y_{1}^{\prime}\right)^{3}}, & -\frac{\partial^{2} F}{\partial u_{\beta} \partial u_{c}} & \frac{y_{\beta}^{\prime}}{\left(y_{1}^{\prime}\right)^{2}} \\
-\frac{\partial^{2} F}{\partial u_{r} \partial u_{\beta}} & \frac{y_{\beta}^{\prime}}{\left(y_{1}^{\prime}\right)^{2}}, & \frac{\partial^{2} F}{\partial u_{r} \partial u_{c}} & \frac{1}{y_{1}^{\prime}}
\end{array}\right|,
$$

where $r, c, \beta, \gamma=2, \cdots, n$ and $\beta$ and $\gamma$ are umbral. The determinant on the right in (5) is easily shown to be identically zero. Hence a problem (1) with an integrand function (4) is always singular.

Assume (1) to be a non-singular problem and restrict attention to that portion of the fundamental region $R$ in which the quantities $f$ and $\left(f-y_{\mu}^{\prime} \partial f / \partial y_{\mu}^{\prime}\right)$ are different from zero. In this subregion of $R$ the ratios

$$
t_{i}=\left(\partial f / \partial y_{i}^{\prime}\right) /\left(f-y_{\mu}^{\prime} \partial f / \partial y_{\mu}^{\prime}\right), \quad(i=1, \cdots, n),
$$

are well defined and of class $C^{\prime \prime}$. These ratios will be called the transversality coefficients of the problem (1).

It is found that

$$
1+y_{\mu}^{\prime} t_{\mu}=f /\left(f-y_{\mu}^{\prime} \partial f / \partial y_{\mu}^{\prime}\right) .
$$

Hence in the subregion of $R$ specified, the transversality coefficients $t_{i}$ necessarily satisfy the inequality

$$
1+y_{\mu}^{\prime} t_{\mu} \neq 0 .
$$

Geometrically this means that the extremal through the point $\left(x, y_{1}, \cdots, y_{n}\right)$ in the direction $1: y_{1}^{\prime}: \cdots: y_{n}^{\prime}$ is not tangent to the hyperplane with normal $1: t_{1}: \cdots: t_{n}$ in this point. 
Finally it is easy to verify that

$$
\begin{array}{r}
\left|\partial t_{i} / \partial y_{j}^{\prime}\right|=\frac{\left|\partial^{2} f / \partial y_{i}^{\prime} \partial y_{j}^{\prime}+t_{i} y_{\mu}^{\prime} \partial^{2} f / \partial y_{\mu}^{\prime} \partial y_{j}^{\prime}\right|}{\left(f-y_{\mu}^{\prime} \partial f / \partial y_{\mu}^{\prime}\right)^{n}}, \\
(i, j=1, \cdots, n) .
\end{array}
$$

The value of the determinant on the right in (9) is given by the product of the determinants $D$ and $\left|\delta_{j}{ }^{i}+y_{i}^{\prime} t_{j}\right|$, the latter of which is found to be equal to $1+y_{\mu}^{\prime} t_{\mu}$. Hence

$$
\left|\partial t_{i} / \partial y_{j}^{\prime}\right|=f D /\left(f-y_{\mu}^{\prime} \partial f / \partial y_{\mu}^{\prime}\right)^{n+1},
$$

and therefore in the subregion of $R$ under consideration the Jacobian of the $t_{i}$ with respect to the $y_{j}^{\prime}$ is different from zero.

3. Conditions for a Set of $n$ Functions to be Transversality Coefficients. The condition (2) establishes a certain relation between the lineal element $\left(x, y_{1}, \cdots, y_{n}, y_{1}^{\prime}, \cdots, y_{n}^{\prime}\right)$ through the point $\left(x, y_{1}, \cdots, y_{n}\right)$ and the hyperplane of directions $\left(d x: d y_{1}: \cdots: d y_{n}\right)$ with normal $\left(1: t_{1}: \cdots: t_{n}\right)$ through this point. If $n$ given functions $T_{i}\left(x, y_{1}, \cdots, y_{n}, y_{1}^{\prime}, \cdots, y_{n}^{\prime}\right)$, $i=1, \cdots, n$, of class $C^{\prime \prime}$ and satisfying the inequalities $1+y_{\mu}^{\prime} T_{\mu} \neq 0, \quad\left|\partial T_{i} / \partial y_{j}^{\prime}\right| \neq 0$ in a region $S$ of $\left(x, y_{1}, \cdots, y_{n}\right.$, $\left.y_{1}^{\prime}, \cdots, y_{n}^{\prime}\right)$-values are to be the transversality coefficients of a non-singular problem (1) in $S$ then the equations

$$
d x+T_{\mu} d y_{\mu 2}=0
$$

and (2) must define the same hyperplane of directions $\left(d x: d y_{1}: \cdots: d y_{n}\right)$ for all sets $\left(x, y_{1}, \cdots, y_{n}, y_{1}^{\prime}, \cdots, y_{n}^{\prime}\right)$ in $S$. Hence corresponding coefficients in these two relations must be proportional. Consequently a set of $n$ functions $T_{i}$ as described can be transversality coefficients of a non-singular problem (1) only in case there exists a function $f\left(x, y_{1}, \cdots, y_{n}\right.$, $\left.y_{1}^{\prime}, \cdots, y_{n}^{\prime}\right)$ satisfying the system of equations

$$
f-y_{\mu}^{\prime} \partial f / \partial y_{\mu}^{\prime}=h, \partial f / \partial y_{i}^{\prime}=h T_{i}, \quad(i=1, \cdots, n),
$$

and different from zero in $S$. In (12) $h \neq 0$ is a function of $\left(x, y_{1}, \cdots, y_{n}, y_{1}^{\prime}, \cdots, y_{n}^{\prime}\right)$. Elimination of $h$ in (12) leads to the following system of non-homogeneous partial differential equations which must be satisfied by $f$ regarded as a function of the $n$ independent variables $\left(y_{1}^{\prime}, \cdots, y_{n}^{\prime}\right)$ and the $n+1$ parameters $\left(x, y_{1}, \cdots, y_{n}\right)$ : 


$$
T_{k} f-\left(\delta_{k}^{\beta}+T_{k} y_{\beta}^{\prime}\right)\left(\partial f / \partial y_{\beta}^{\prime}\right)=0, \quad(k=1, \cdots, n) .
$$

To determine the integrability conditions for the system (13) we transform it into a system of equations linear and homogeneous in the first partial derivatives of a function $G\left(x, y_{1}, \cdots, y_{n}, y_{1}^{\prime}, \cdots, y_{n}^{\prime}, f\right)$ with $G_{f} \neq 0$ which defines $f$ by means of the equation $G=$ constant. The resulting system is

$$
U_{k} G=\left(\delta_{k}^{\beta}+T_{k} y_{\beta}^{\prime}\right)\left(\hat{c} G / \partial y_{\beta}^{\prime}\right)+T_{k} f(\hat{c} G / \partial f)=0 .
$$

The matrix of coefficients of (14) contains the $n$th order determinant $\left|\delta_{k}^{i}+y_{i}^{\prime} T_{k}\right|, i, k=1, \cdots, n$, which has the value $\left(1+y_{\mu}^{\prime} T_{\mu}\right)$ and hence is different from zero in $S$. Since the $n$ equations in (14) are therefore independent, it follows that if a single one of the commutators of this system is not a linear combination of the original equations the only solution for $G$ is a constant and hence no solution $f$ of (13) exists. Therefore the equations (13) are compatible if and only if the system (14) is a complete system.

A typical commutator of the system (14) is found to have the value

(15) $\left(U_{m} U_{k}\right) G=\left(\Gamma_{m k} y_{\beta}^{\prime}+T_{k} \delta_{m}^{\beta}-T_{m} \delta_{k}^{\beta}\right)\left(\partial G / \partial y_{\beta}^{\prime}\right)+\Gamma_{m k} f(\partial G / \partial f)$,

where

(16) $\Gamma_{m k}=y_{\nu}^{\prime}\left(T_{m} \partial T_{k} / \partial y_{\nu}^{\prime}-T_{k} \partial T_{m} / \partial y_{\nu}^{\prime}\right)+\left(\partial T_{k} / \partial y_{m}^{\prime}-\partial T_{m} / \partial y_{k}^{\prime}\right)$.

In order that

$$
\left(U_{m} U_{k}\right) G=r_{\nu} U_{\nu} G, \quad m, k=1, \cdots, n, \quad m<k,
$$

where the $r_{i}(i=1, \cdots, n)$ are coefficients of combination it is necessary that

$$
\begin{aligned}
\Gamma_{m k} y_{i}^{\prime}+T_{k} \delta_{m}{ }^{i}-T_{m} \delta_{k}{ }^{i} & =r_{\nu}\left(\delta_{\nu}{ }^{i}+T_{\nu} y_{i}^{\prime}\right), \quad i=1, \cdots, n, \\
\Gamma_{m k} f & =r_{\nu} T_{\nu} f .
\end{aligned}
$$

Since $f \neq 0$ it follows that

$$
\begin{aligned}
& r_{i}=0 \text { if } i \neq m, k, \\
& r_{i}=T_{k} \text { if } i=m, \\
& r_{i}=-T_{m} \text { if } i=k .
\end{aligned}
$$

Hence the $n(n-1) / 2$ quantities $\Gamma_{m k}$ are necessarily zero if (14) is a complete system. Conversely the conditions 


$$
\Gamma_{m k}=0, m, k=1, \cdots, n, m<k,
$$

are sufficient conditions for the system (14) to be complete, for if all of the $\Gamma_{m k}$ vanish then there actually exist coefficients $r_{i}$, namely those in (19), for which the relations (17) subsist. Taken in connection with the results of $\$ 2$ this proves the following theorem.

TheOREM 1. Necessary and sufficient conditions for $n$ functions $T_{i}\left(x, y_{1}, \cdots, y_{n}, y_{1}^{\prime}, \cdots, y_{n}^{\prime}\right)$ to be the transversality coefficients of a non-singular problem (1) in a region $S$ of $\left(x, y_{1}, \cdots, y_{n}, y_{1}^{\prime}, \cdots, y_{n}^{\prime}\right)$-values are that in $S$ the functions $T_{i}$ be of class $C^{\prime \prime}$ and satisfy the inequalities $\left|\partial T_{i} / \partial y_{j}^{\prime}\right| \neq 0$, $1+y_{\mu}^{\prime} T_{\mu} \neq 0$ and the $n(n-1) / 2$ relations $(20)$.

4. Determination of the most general Integrand Function. Suppose that $n$ functions $T_{i}$ which satisfy the conditions of Theorem 1 are given. What is the most general integrand function $f\left(x, y_{1}, \cdots, y_{n}, y_{1}^{\prime}, \cdots, y_{n}^{\prime}\right)$ of a non-singular problem (1) which has the set $T_{i}$ as its transversality coefficients? To answer this question consider the line integral

$$
H=\int_{0}^{y^{\prime}} T_{\mu} d y_{\mu}^{\prime} /\left(1+y_{\mu}^{\prime} T_{\mu}\right)
$$

in the $\left(y_{1}^{\prime}, \cdots, y_{n}^{\prime}\right)$-space. In (21) the symbols 0 and $y^{\prime}$ used as limits represent $n$-partite variables. It is found that necessary conditions for (21) to be independent of the path of integration are that the $T_{i}$ satisfy the $n(n-1) / 2$ relations*

$$
R_{i l}=0, i, k=1, \cdots, n, \quad i<k,
$$

where

$$
\begin{aligned}
& R_{m k}=y_{\mu}^{\prime}\left[T_{m} \partial T_{\mu} / \partial y_{k}^{\prime}-T_{k} \partial T_{\mu} / \partial y_{m}^{\prime}\right. \\
& \left.\quad+T_{\mu}\left(\partial T_{k} / \partial y_{m}^{\prime}-\partial T_{m} / \partial y_{k}^{\prime}\right)\right]+\left(\partial T_{k} / \partial y_{m}^{\prime}-\partial T_{m} / \partial y_{k}^{\prime}\right) .
\end{aligned}
$$

These conditions are also sufficient conditions for independence of path in a space of suitably simple connectivity properties.

It can be shown that in terms of the $\Gamma_{m k}$ introduced in (16)

$$
R_{m k}=\left(1+y_{\mu}^{\prime} T_{\mu}\right) \Gamma_{m k}+y_{\mu}^{\prime} T_{k} \Gamma_{\mu m}+y_{\mu}^{\prime} T_{m} \Gamma_{k \mu} .
$$

* Compare Rawles, loc. cit., p. 778. 
Hence when all of the $\Gamma_{m k}$ vanish, as they do for the set $T_{i}$ under consideration, the conditions for the line integral $H$ to be independent of the path in such a space are satisfied.

A particular solution of the homogeneous system (14) is

$$
G=f e^{-H} .
$$

Hence, since the general integral of a complete system of $n$ equations in $n+1$ independent variables is an arbitrary function of a single particular integral of the system, ${ }^{*}$ the most general solution of (14) is given by

$$
G=F\left(f e^{-H} ; x, y_{1}, \cdots, y_{n}\right),
$$

where $F$ is an arbitrary function of the arguments indicated. The most general non-singular solution $f$ of the system of equations (13) is now obtained by solving for $f$ the equation $F\left(f e^{-H} ; x, y_{1}, \cdots, y_{n}\right)=0 . \dagger$ Hence we reach the following conclusion.

Theorem 2. If $n$ functions $T_{i}\left(x, y_{1}, \cdots, y_{n}, y_{1}^{\prime}, \cdots, y_{n}^{\prime}\right)$ satisfy the conditions of Theorem 1 , then there always exists a non-singular problem (1) with an integrand function $f$ of class $C^{\prime \prime \prime}$ which has the functions $T_{i}$ as its transversality coefficients. The most general such problem has an integrand function

$$
f=g\left(x, y_{1}, \cdots, y_{n}\right) e^{H}
$$

where $g$ is different from zero and of class $C^{\prime \prime \prime}$ but is otherwise an arbitrary function of its arguments, and where $H$ has the value (21).

An integrand function $f$ of the form (27) was found in an entirely different manner by Rawles. His argument although of much interest requires the decidedly restrictive hypothesis of the existence of an $n$-parameter family of Mayer fields; and, moreover, does not show that a function $f$ of the form (27) is actually the most general integrand function of a problem (1) with a prescribed transversality condition.

The University of Chicago

* Goursat, Leçons sur l'Intégration des Équations aux Dérivées Partielles du Premier Ordre, 1921, p. 71.

$\dagger$ Goursat, loc. cit., p. 95. 